\title{
A Novel Method and Implementation of Digital Algorithm for First Order Non Linear Spherical Tank Level Control Process with an Integral Windup
}

\author{
R. Kiruba, K.Malarvizhi
}

\begin{abstract}
The primary goal of the process control is to control, monitor and to maintain different kinds of process stations at the desired stable conditions for improving the productivity and product quality. In industries most of the process are non - linear, controlling such type of system is difficult. So, spherical tanks are one among the non-linear systems which can be used to store maximum quantity of product in minimum area. These types of non-linear systems violate the superposition principle and are very difficult to control and monitor. Generally, conventional PID Controller is used for controlling a process in a plant. In Level control process, there exists the sudden change in final control element which leads to maximum overshoot and larger settling time, this stated phenomenon requires tuning of gain values to control the process variable to the set point. In order to overcome the above-mentioned problem, Anti reset windup concept is preferred. The mathematical modelling of a given process is obtained using black box system identification methodology. The accuracy in controlling the process variable is complicated due to its dynamic behaviour and characteristics. So in order to maintain the proper characteristics of non-linear system the mathematical modelling has been obtained by conducting the open loop test. In ancient stage the Ziegler Nicholas method is implemented to tune the PID parameters such as $K p$, $\tau$ i and $\tau d$ which takes larger settling time, maximum Integral Square error and maximum peak overshoot. After tuning the PID gain values with the help of anti reset windup, the overshoot and settling time will be reduced. In this proposed work, Deadbeat and Dahlin's Algorithms are used to convert the transfer function of the level process from ' $S$ ' domain to ' $Z$ ' domain (digital controller). The transfer function is used in MATLAB (Matrix Laboratory) to analyse the output of level process and the simulations are taken for the plant with above mentioned two different algorithms. For real time interface the LabVIEW platform is used and the results are discussed. This proposed digital controller with anti reset windup provides lower steady state error, minimum peak overshoot and minimum error criteria indices.
\end{abstract}

Index Terms-Spherical tank, Integral windup, PID, Deadbeat, Dahlin's algorithm, MATLAB, LabVIEW.

\section{INTRODUCTION}

Nowadays, it is difficult to satisfy the requirements of the process plants and its been increasing. In order to improvise the product quality specifications and productivity, it requires to concentrate more on safety aspects,rapid changes in process environment and cost involvement. In the olden day the measurement of level itself is a tedious process because of lagging in technology development.[1][2] They use simple instruments like sight glass indicator, floats, bubble indicators and level switches for the measurement of

level. Differential pressure transmitter plays an important role in the measurement of level of non-linear systems. The real challenges in process control is to control the level of a tank which has nonlinear characteristics because it has large settling time and non-linear shape.[4]

Since PID control has greatest advantages and efficiency over other control actions, it has been adopted widely to control most of the industrial automation processes. A PID controller will take corrective action based on the error calculated and tries to adjust the process to operate in its stable operating range. Open loop test gives a transfer function for level process and simulated using PID controller. Here Deadbeat and Dahlin's Algorithms are used to convert the transfer function of the level process from ' $S$ ' domain to ' $Z$ ' domain.[3] The transfer function is used in MATLAB to analyze the output of level process and the simulations are taken for the plant with above mentioned two different algorithm. Each simulation were compared and analyzed to get less settling time and reduced peak overshoot.

\section{EXISTING METHODOLOGY}

In feedback control theory, Proportional Integral and Derivative (PID) control algorithm is the most basic control algorithm used often, because of its remarkable efficacy, simplicity of implementation and broad applicability. Since PID has its remarkable advantages such as ease in practical use and proficient working dynamics are some of the pivotal reasons behind the large acceptance of the PID control. The control of level in such non-linear tanks can be achieved with the help of modeling and simulation. Initially open loop test is conducted for a particular level and the transfer function is obtained with the help of Ziegler Nicholas method. From the mathematical modeling the transfer function of controller parameters are tuned by using Ziegler Nicholas method for the process to settle at the specified set point. The genetic algorithm and different algorithms were used for controlling the plant.

\section{A. About PID}

The mathematical representation of PID Controller is,

$$
\mathrm{u}(\mathrm{t})=\mathrm{MV}(\mathrm{t})=\mathrm{K}_{\mathrm{p}} \mathrm{e}(\mathrm{t})+\mathrm{K}_{\mathrm{i}_{0}}^{\mathrm{t}} \mathrm{e}(\mathrm{t}) \mathrm{dt}+\mathrm{K}_{\mathrm{d}} \frac{\mathrm{d}}{\mathrm{dt}} \mathrm{e}(\mathrm{t})-\mathrm{-}^{-(1)}
$$

Revised Manuscript Received on 14 August, 2019.

Mrs. R. Kiruba, Assistant Professor/EIE, SNS College of Technology, Coimbatore, Tamilnadu, India.

Dr.K.Malarvizhi, Professor/EEE, Kumaraguru College of Technology, Coimbatore, Tamilnadu, India.

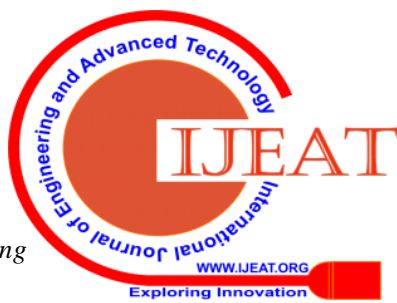




\section{A NOVEL METHOD AND IMPLEMENTATION OF DIGITAL ALGORITHM FOR FIRST ORDER NON LINEAR SPHERICAL TANK LEVEL CONTROL PROCESS WITH AN INTEGRAL WINDUP}

As in the equation (3.1) the derivative action (also called rate or pre-act) anticipates where the procedure is headed by looking at the time rate of change of the controlled variable i.e. its derivative. $\mathrm{Td}$ is the 'rate time' and this characterizes the derivative position with units of minutes. In theory derivative action should always progress dynamic response and it does in many loops. In others, nevertheless, the problem of noisy signals makes the use of derivative action undesirable differentiating noisy signals can translate into disproportionate MV movement. Derivative action depends on the slope of the error, unlike P and I. If the error is constant derivative action has no effect.

\section{B. Level control using PID}

Level control of a spherical tank is a complex issue because of the nonlinear nature of the tank. For each stable operating point, a First Order Process with Time Delay model was identified using process reaction curve method. The need for improved performance of the procedure has led to the progress of the finest controllers. The level in spherical tank can be controlled by PID controller and it can be monitored. Initially step response is given to the error detector and it gives difference between the set point and feedback values.

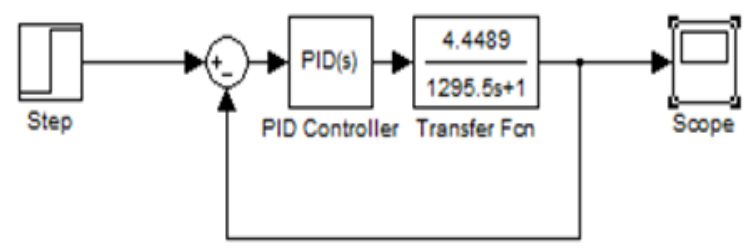

Fig. 1. Level control using PID

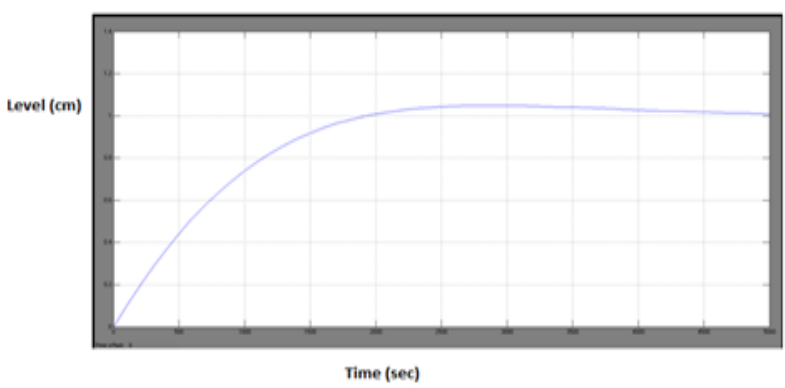

Fig. 2. Output for the Level Control Using PID

PID takes action according to the error values. Level can be controlled by varying or tuning the PID gain values using Ziegler Nicholas method.

\section{PROPOSED METHODOLOGY}

Typically by a PLC (Programmable Logic Controller), an intelligent device which is used to devise the controller for a sampled data control systems. The PLC attempts to realise the controller algorithm, the signals used for process of the controller (Noise reduction, A/D and D/A converters). It has extension to apply different and more sophisticated control algorithm, as the controller algorithm is developed by software. One of these algorithms confirms the perfect settling of the output signal during a limited bound of time, limited custom of the sampling periods. As mentioned in the literature survey, this algorithm is referred to as dead-beat controller algorithm.[4] To comprehend the fundamental nature of the method - for simplicity - think about a stable plant without dead time. A standard unit step is used as a reference signal. (Let us remark that the dead-beat controller can be designed in a bit more complex way by taking into account unbalanced plants with dead time, a reference signal different of unit step as well). The design process is derived in three promiting steps. This solution satisfify the realistic necessities. In the first step the controller is designed for the fastest performance when the output signal is settled in one sampling step. It will be seen that with this aim with distinctive sampling times the control signal could be enormously high, in addition in most situvations oscillations take place between the sampling points. In the second step the design is better to stay away from intersampling oscillations.

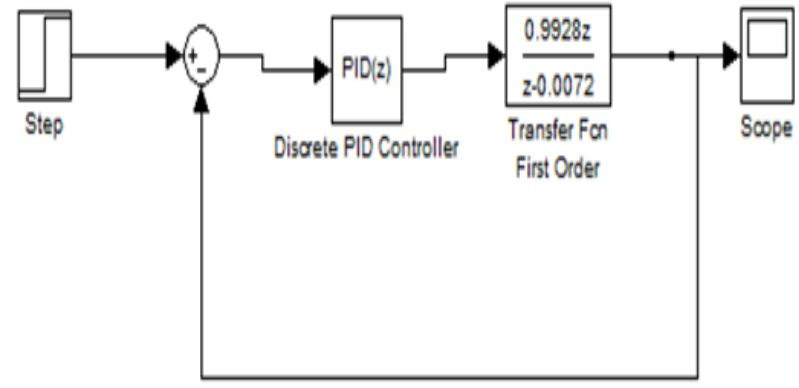

Fig. 3. Block Diagram of Deadbeat Algorithm

\section{A. Deadbeat Algorithm Implementation}

It will cause oscillations when there is a termination of zeros exterior the unit circle. Zeros of the plant and transfer function (TF) are alienated for non cancellable and cancellable ones and cancellable zeros only will become visible in the controller program. This type of adaptation in the algorithm increases the settling time. If the control signal is still advanced than tolerable confines, the solution can be tuned using a so-called design polynomial. If this pretains to be, the settling time is improved further (but still remains finite). The devise is executed in the ' $z$ ' operator field being discrete. Interesting attribute of the design procedure is the reality that it eliminates detrimental time field properties (oscillations, too high values of the control signal) by statement done in the ' $z$ ' operator domain. The design of a sampled data controller is the basic task.

$$
\begin{aligned}
& G(s)=\frac{4.4489}{1295.5 s+1}(1) \\
& G_{h 0}(s)=\frac{1-e^{-T s}}{s}
\end{aligned}
$$

Let us assume the $\mathrm{T}$ value of Zero Order Hold is 1 $(\mathrm{T}=1)$,

$$
\begin{aligned}
& Z\left\{G(s) G_{h 0}(s)\right\}=Z\left\{\frac{1-e^{-T s}}{s} \cdot \frac{4.4489}{1295.5 s+1}\right\}(3) \\
& G(z)=\left(1-z^{-1}\right) Z\left\{\frac{4.4489}{s(1295.5 s+1)}\right\}(4)
\end{aligned}
$$

By applying partial fraction,

$$
\frac{4.4489}{s(1295.5 s+1)}=\frac{A}{S}+\frac{B}{1295.5 s+1}
$$$$
4.4489=A(1295.5 s+1)+B s
$$ 
Put $\mathrm{s}=0$ then, $\mathrm{A}=4.4489$ and put $\mathrm{s}=\frac{-1}{1295.5}$ then, $\mathrm{B}=-$ 5763.55. Substitute the values of $A$ and $B$ in Equation 5.1.

$$
\begin{gathered}
G(z)=\frac{z-1}{z} Z\left\{\frac{4.4489}{s}-\frac{5763.55}{1295.5 s+1}\right\} \\
G(z)=(4.4489) \frac{z-1}{z}\left\{\frac{z}{z-1}-\frac{z}{z-e^{-0.0072 * 1}}\right\} \\
G(z)=4.4489\left\{\frac{z-0.9928-z+1}{z-0.9928}\right\} \\
G(z)=\frac{0.032}{z-0.9928}
\end{gathered}
$$

The DeadBeat eqution is,

$$
D(z)=\frac{1}{G(z)} \frac{z^{-1}}{1-z^{-1}}
$$

By substituting the equation 5.2 in $\mathrm{D}(\mathrm{z})$, then

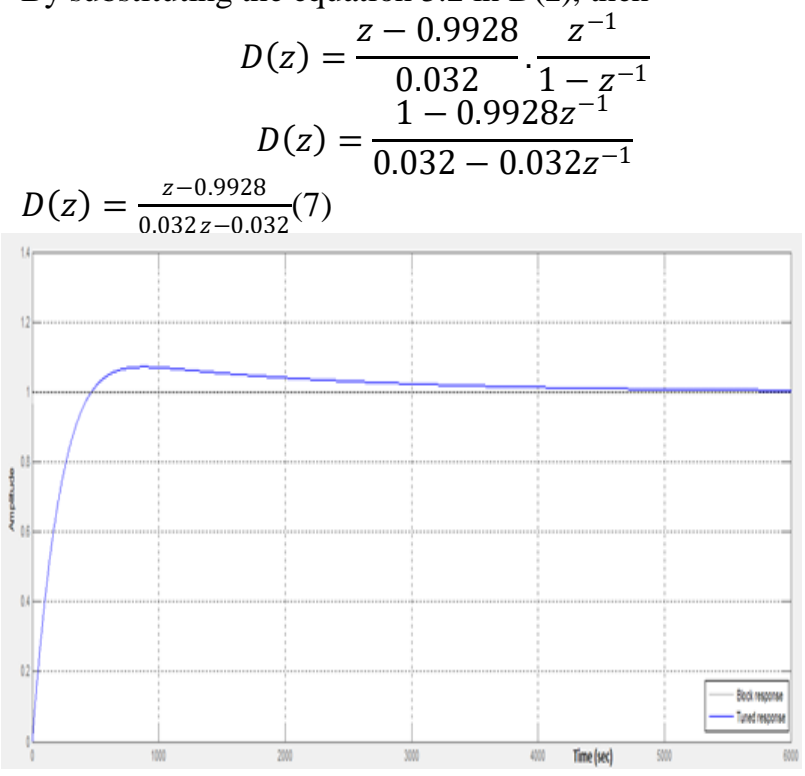

Fig. 4. Simulation Response for Deadbeat Algorithm

The equation (7) is the deadbeat controller for the nonlinear level procedure. It is pretended in the MATLAB by auto tuning the PID parameters and the response reached the set point rapidly by using deadbeat algorithm.

\section{B. Implementation of Dahlin's Algorithm}

Dahlin's algorithm has a major difficulty which cause a steady-state fault by an input-side interruption for an integrator plant with time delay. To notch, it has difficulty which will not ensure follow-up action to a response model by parameter error of controller. To solve these problems, here just introducing discrete-time IMC and iterative least squares procedure. The block of Dahlin's algorithm is shown in fig 5

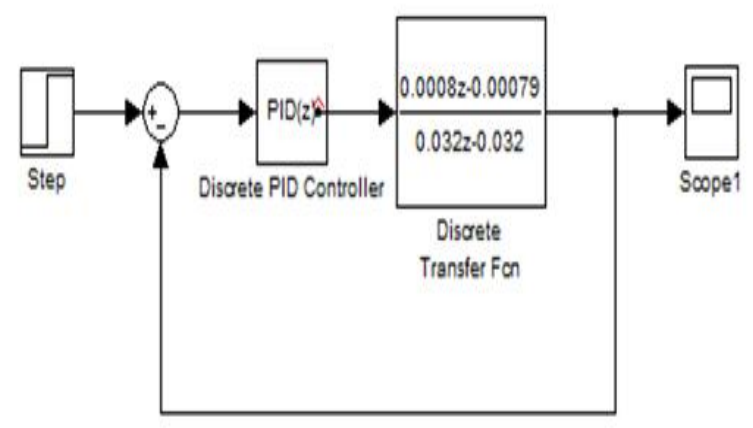

Fig. 5 Block Diagram of Dahlin's Algorithm
The Dahlin's Equation is,

$$
\begin{gathered}
D(z)=\frac{1}{G(z)} \frac{(1-\propto) z^{-N-1}}{1-\propto z^{-1}-(1-\propto) z^{-N-1}}(8) \\
D(z)=\frac{z-0.9928}{0.032} \cdot \frac{(1-\propto) z^{-N-1}}{1-\propto z^{-1}-(1-\propto) z^{-N-1}} \\
\theta d=N T \text { Tand } \propto=e^{-\frac{T}{\tau}}
\end{gathered}
$$

Where $\mathrm{T}=1$ and $\theta d=0$, then $\mathrm{N}=0$

$$
\begin{gathered}
\propto=e^{-\frac{T}{\tau}}=e^{-\frac{1}{1295.5}} \\
\propto=0.9992
\end{gathered}
$$

Substitute the values of $\propto$ and $\mathrm{N}$ in above $D(z)$ equation

$$
\begin{gathered}
D(z)=\frac{z-0.9928}{0.032} \cdot \frac{(1-0.9992) z^{-1}}{1-0.9992 z^{-1}-(1-0.9992) z^{-1}} \\
D(z)=\frac{z-0.9928}{0.032} \cdot \frac{0.0008 z^{-1}}{1-z^{-1}} \\
D(z)=\frac{\left(1-0.9928 z^{-1}\right) 0.0008 z^{-1}}{0.032 z^{-1}-0.032 z^{-2}} \\
D(z)=\frac{0.0008-0.00079 z^{-1}}{0.032-0.032 z^{-1}} \\
D(z)=\frac{0.0008 z-0.00079}{0.032 z-0.032}(9)
\end{gathered}
$$

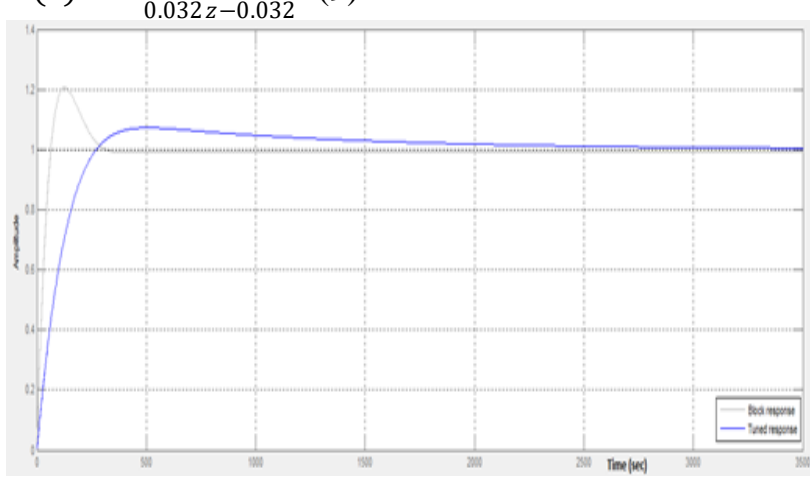

Fig. 6. Simulation Response for Dahlin's Algorithm

The equation 8 is the dahlin's controller for the non-linear level process. It is simulated in the MATLAB by auto tuning the PID parameters and the response reached the set point soon by using dahlin's algorithm.

\section{OPEN LOOP TEST IN SPHERICAL PLANT}

\section{A. Procedure For Open Loop Test}

- In the open loop test the outflow and the discharge pump speed is held at a constant value.

- The inflow to the spherical tank is manipulated by varying the current to control the inflow control valve.

- The current 4 to $20 \mathrm{~mA}$ to I / P converter is given accurately by using 6211 USB DAQ through PC with LabVIEW software.

- The output of the USB 6211 DAQ Card is usually 0 to $5 \mathrm{~V}$ and it is converted into 4 to $20 \mathrm{~mA}$ current signals by using $\mathrm{V}$ to I converter.

- The bypass or pressure relief valve of the pump is also maintained at constant position throughout the process. For this the system input and output is maintained constant and the system is allowed to settle by own self regulation 


\section{A NOVEL METHOD AND IMPLEMENTATION OF DIGITAL ALGORITHM FOR FIRST ORDER NON LINEAR SPHERICAL TANK LEVEL CONTROL PROCESS WITH AN INTEGRAL WINDUP}

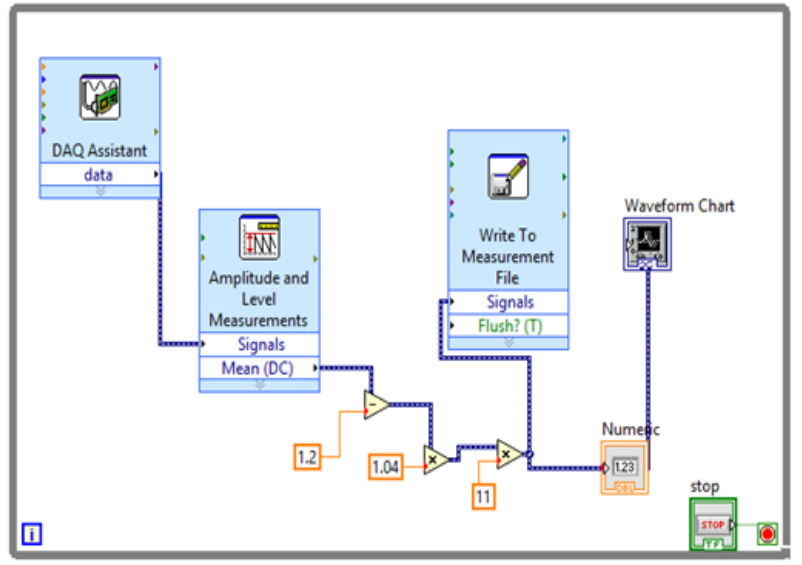

Fig. 7.Open loop test using LabVIEW

Open loop test was done by running the spherical plant using LabVIEW program. The level of the spherical tank is measured by connecting the Level Transmitter to the DAQ

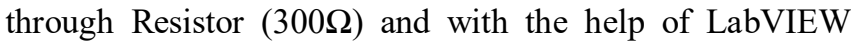
program the values are noted. The input to the control valve is varied as $2.0 \mathrm{~V}, 2.1 \mathrm{~V}$ and $2.2 \mathrm{~V}$ when set point is $15 \mathrm{~cm}$, $30 \mathrm{~cm}$ and $50 \mathrm{~cm}$.

\section{HARDWARE INFORMATION}

\section{A. The Experimental setup}

The experimental set up for this system consists of a spherical tank and the reservoir.

The pump with the water through the upstream to the spherical tank and there is a Pressure Relief Valve (PRV) to avoid damage to the pump in no load conditions. The upstream measuring elements include rotameter and Orifice type Flow Transmitter (FT) for flow measurement and the actuating elements are inflow pneumatic control valve (CV1) and hand valves (HV1 and HV2). The pneumatic manage valve is air to open and manipulates the flow of the liquid pumped to the spherical tank from the liquid reservoir. The level of the liquid in the tank is deliberate by means of the DPT Level Transmitter (LT). In the downstream the flow is controlled by outflow pneumatic organize valve (CV2) and hand valve (HV3). An electro pneumatic converter (I/P Converter) provides necessary pneumatic signals to actuate the control valves. Fig. 8.shows the real time investigational setup of a spherical tank.

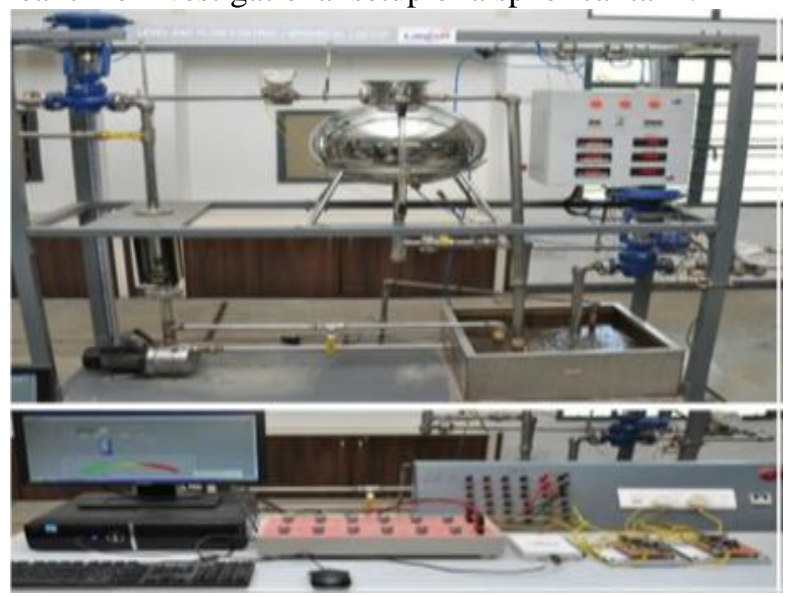

Fig. 8 Experimental setup

\section{RESULT ANALYSIS}

In this proposed work, non-linear process (level) transfer function was derived and analyzed with different algorithms. The output of different algorithm was taken with PID controller and analyzed. Integral windup is more efficient when compared to normal PID because it reduces the overshoot and settle the process variable in set point with less time. The output of level process transfer function using PID was analyzed and the transfer function is converted into discrete form by using the deadbeat and dahlin's algorithms which are simulated by using the discrete PID. The settling time for ' $S$ ' domain level transfer function and ' $\mathrm{Z}$ ' domain transfer function are compared. The time domain specifications for normal PID, Dead Beat algorithm and Dahlin's algorithm are shown in figure $9,10 \& 11$

\begin{tabular}{|lll|} 
& Tuned & Block \\
\hline Rise time (sec) & 981 & 18.1 \\
Settling time (sec) & $3.46 \mathrm{e}+003$ & $1.83 \mathrm{e}+003$ \\
Overshoot $(\%)$ & 6.77 & 89.3 \\
Peak & 1.07 & 1.89 \\
Gain margin (db @ rad/sec) & Inf @ Inf & Inf @ NaN \\
Phase margin (deg @ rad/sec) & $63.9 @ 0.001 \ldots 4.11$ @ 0.0587 \\
Closed-loop stability & Stable & Stable \\
& &
\end{tabular}

Fig 9 Time domain specifications for Level Process Using Normal PID

\begin{tabular}{|lll|} 
& Tuned & Block \\
\hline Rise time (sec) & 320 & 0 \\
Settling time (sec) & $3.27 \mathrm{e}+003$ & 2 \\
Overshoot (\%) & 7.17 & 0.0226 \\
Peak & 1.07 & 1 \\
Gain margin (db @ rad/sec) & - Inf @ 0 & - Inf @ 0 \\
Phase margin (deg @ rad/sec) & $80.6 @ 0.005 . .$. Inf @ NaN \\
Closed-loop stability & Stable & Stable \\
& & \\
& & \\
& & \\
& & \\
\end{tabular}

Fig 10 Time domain specifications for Level Process Using Deadbeat Algorithm

\begin{tabular}{lll}
\hline & Tuned & Block \\
\hline Rise time (sec) & 187 & 48 \\
Settling time (sec) & $1.91 \mathrm{e}+003$ & 278 \\
Overshoot (\%) & 7.25 & 20.7 \\
Peak & 1.07 & 1.21 \\
Gain margin (db @ rad/sec) & - Inf @ 0 & - Inf @ 0 \\
Phase margin (deg @ rad/sec) & $80.1 @ 0.009$ & $66.1 @ 0.0273$ \\
Closed-loop stability & Stable & Stable
\end{tabular}

Fig 11 Time domain specifications for Level Process Using Dahlin's Algorithm 
In above, the settling time for normal PID is 70 seconds. The settling time for discrete PID using deadbeat algorithm is 65 seconds and using dahlin's algorithm is 38 seconds. The Dahlin's algorithm is more efficient to analyze and control the level process when compared to normal PID and discrete PID using deadbeat algorithm.

\section{CONCLUSION AND FUTURE WORK}

In this proposed work, the response of a nonlinear system model was implemented using LabVIEW and the required test was done to get the respective transfer function. The transfer function(TF) was obtained from the open loop test values and MATLAB System Identification Tool Ziegler Nichols tuning methodology. The Ziegler Nichols tuning method is used to obtain the tuning parameters for obtaining smooth response without transient outputs. The implementation of Deadbeat and Dahlin's algorithm for plant transfer function allows an effective way of functioning. It has more strong stability, effectiveness and can solve the tuning problems of PID controller parameters more without difficulty and rapidly than the existing methods.

\section{REFERENCES}

1. C. Bohn and D. P. Atherton. "An analysis package comparing PID ant windup strategies", IEEE Systems Magazine, 5(2):34-40, April 1995.

2. G. J. Silva, A. Datta, and S. P. Bhattacharyya, "PID controllers for time-delay systems," Birkhauser Boston, 2004.

3. [3]

S.Janarthanan,K.N.Thirukkuralkani\&S.Vijayachitra,

"Performance Analysis of Non-Integer Order PID Controller for Liquid Level Control of Spherical Tank System”, IEEE Systems Magazine, ICICES2014.

4. Nisi, K., B. Nagaraj, and A. Agalya. "Tuning of a PID controller using evolutionary multi objective optimization methodologies and application to the pulp and paper industry." International Journal of Machine Learning and Cybernetics (2018): 1-11.

5. Adolf Hermann Glattfelder AndWalter Schaufelberger. "Stability Analysis of Single Loop Control Systems with Saturation and Antireset-Windup Circuits",IEEE Transactions On Automatic Control, Vol. AC-28. No 12. December 1983.

6. R.Valarmathi, P.R.Theerthagiri, S.Rakeshkumar. "Design and Analysis of Genetic Algorithm Based Controllers for Non Linear Liquid Tank System",IEEEInternational Conference On Advances In Engineering, Science And Management (ICAESM -2012) March 30 , 31,2012 . 